\title{
Crowdsourcing: Using real-time social media data to repopulate the socially distanced world
}

\author{
lan Willcock \\ University of Hertfordshire \\ Hatfield, United Kingdom \\ i.willcock@herts.ac.uk
}

\begin{abstract}
The paper suggests that one of the aspects of the pre-Covid world that is most missed in the "new normal' is the social experience of crowds and that immersive media might compensate for this. The nature of crowds is explored with a view to identifying the parameters for successful crowd simulation. There is a brief survey of computer crowd simulation which identifies some of the challenges together with descriptions of some recent games which feature crowds as a significant element. The paper then describes a method using real-time searches of social media content derived from Twitter as an extended data set which can be used to produce variety in the individual agents making up generated crowds and so provide a closer simulation of the crowd experience.
\end{abstract}

\section{Digital art. Data visualisation. Generative digital art. Virtual reality. Collective thought processes}

\section{INTRODUCTION: LIVING IN THE SOCIALLY- DISTANCED WORLD}

The project described in this paper began from the experience of lockdown; the experience common to almost all of us at points over the last year where almost all contact with others is stopped and social isolation, being cut off from the stimulations and provocations of others, becomes the new 'normal'. Networked communications systems, Zoom and Teams became ubiquitous and tried to fill this void, but even short experiences made it clear that while face to face communication with one or a small number of people could be conducted fairly successfully, as the number of participants in an environment increased, the rendering of that crowd experience became increasingly less comfortable. Seeing a number of small video feeds on a screen is simply a very different experience to that of being in a large group of people; what can be effective with small numbers does not scale. If this 'crowd experience' was not important, this lack would not matter, but as the pandemic has continued, it is very clear looking at the evidence of cultural production in the news and social media that one of the most upsetting aspects of the 'new normal' was the lack of crowd experience (Moore 2020). People remarked in melancholic terms about the empty streets, on how they missed going to amiably crowded places (restaurants, bars, performances, galleries).
The use of VR and AR to create or enrich environments has been commonplace for some time but given the situation we were presented with, the need for population became more pressing. The work to be presented is a part of a longstanding project using metadata and dynamically sourced materials to produce digital artworks which reflect real-time aspects of mass, thought. In contrast to previous art works (You. Here. Now. 2013, Ways of Seeing 2015), the most recent project focussed on the processes underlying the emergence of collectively held views - the individual activity associated with presenting a range of polemical positions which are considered in a social, sometimes adversarial process and which leads to some level of consensus. It is perhaps not a coincidence that enforced physical separation has forced these processes to operate in primarily digital spheres; where we once had meetings, protests and seminars, we now have Twitter!

This paper presents a generative VR system called 'The Wisdom of Crowds' which allows the user to choose an issue and then walk through an immersive scene populated by a crowd of placardholding individuals, each given an individual identity and narrative based on a Tweeter (using data mined from Tweet metadata) who has posted on the topic. In a locked down world, the intention is that one can re-experience virtually the excitement and 
fascinating unpredictability associated with crowds; occasions where mass physical embodiment and varying views both contribute to a sense of social connection and the emergence of shared meaning.

\section{THE DIMENSIONS OF CROWDS}

If one is to consider how digital media might satisfyingly repopulate the socially distanced world, one needs to begin by identifying the features of the missing 'crowd-experience', what is a crowd and what are those specific features that are missed?

The obvious answer is that a crowd is simply a large number of people, but this is an oversimplification as there are collections of large numbers of people which are not experienced in the same way; identically dressed and similarly moving people (armies, performers), people standing or sitting completely still (audiences), and collections of people with a single, shared planned purpose (often combined with a shared direction of travel) - queues, marches or protests.

One can identify some of the parameters for the kinds of crowd experiences missing in the locked down world; in a pleasurable, social crowd, participants must exhibit at least some activity but not in an obviously similar or coordinated way, they must be heterogeneous and overall, the 'crowd' should have no obvious, planned, common purpose - individuals' own narratives and purposes should be more prominent that any overall group focus.

We thus identify a three-dimensional parameter space for classifying the relationships between individual participants and the overall crowd; activity, appearance and narrative. Each of these qualities can be mapped on an axis representing a scale from totally similar (i.e. each member of the crowd is doing, appearing or following the same) to totally different, see Figure 1 . The different collections of people mentioned above would all occupy slightly different places within this parameter space. The position of a crowd in this space is dynamic rather than static, crowds may change their location on the activity and narrative axes in response to external events such as an emergency or an event needing to be celebrated.

In addition to the qualitative dimensions of crowds, they also have a quantitative aspect. There is an obvious lower limit on the number of people required for a 'crowd', it must be more than two (there is a popular saying in English, "Two is company but three is a crowd" which might suggest that there need to be at least three people for a crowd to form, however I would suggest that this conclusion would be to misunderstand the intention behind the saying - which is far more about the desire for the two to be

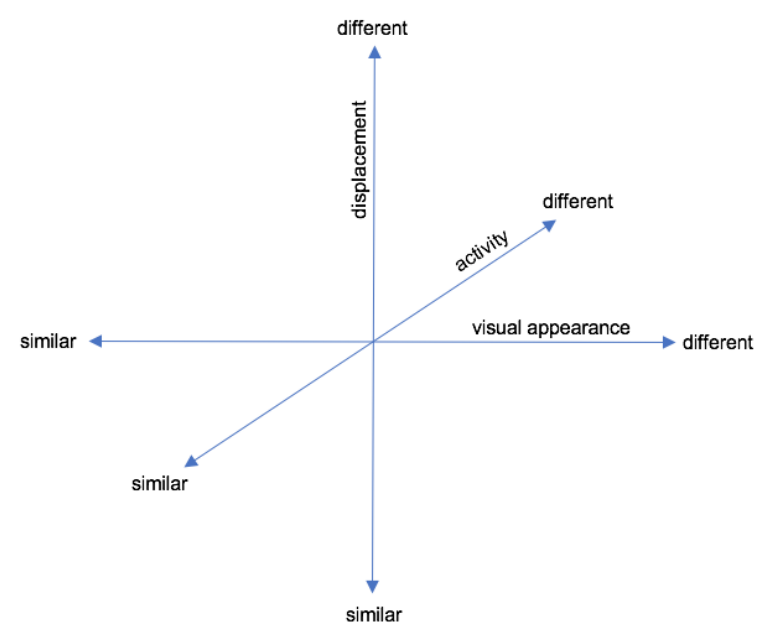

Figure 1: the Crowd:Individual parameter space

alone with each other than to specify the dimensions of crowds), but how many more? There are actually two numbers that are important here; the absolute number of participants and (more importantly) the 'crowd density'; fifty people may be perceived as a crowd in a small enclosed space but not if distributed more or less evenly across a wide area such as a park. There is also some evidence (Filingeri et al 2017; Dickinson et al 2019) that crowd density is a critical parameter in the individual's perception of the crowd experience; if we are closely surrounded (so that movement and agency are becoming restricted by the proximity of others), the experience becomes less pleasurable and may become frustrating or even frightening, "Unwelcome close proximity to other people and a lack of sufficient personal space in a crowded environment were commonplace negative experiences" (Filingeri et al 2017). Dickinson et al (2017) provide persuasive evidence that the effects of real-world proxemics map closely to those in virtual reality.

\section{SIMULATING CROWDS}

Crowd simulation has been a hot topic in computer science since the late 1980s (Thalmann, \& Musse 2012:11) and, because of the wide range of potential applications, remains a popular area. From this extensive body of work:

"many crowd simulation methodologies have been developed to model crowd movement, which can be roughly classified into macroscopic (continuum-based) and microscopic (agentbased) models" (Xu et al 2014).

For many applications (safety planning etc.) the overall behaviour of the crowd is the most important factor - visualisation may be symbolic or use simple 2D approximations, but the crowd behaviour may be modelled using sophisticated, 
psychologically-informed algorithms validated against observational data.

For visualisation-focussed applications such as games and immersive media (particularly given their frequent need to render environments that are not based on observable realities), there is a requirement for both the appearance of the crowd and any interaction with an individual in that crowd to be convincing. This means that individuals need to be implemented as sets of agents, but the qualities previously identified in (convincing) crowds, heterogeneity of appearance and activity, semi-random but individually purposeful movement, make large numbers of crowd members computationally expensive to generate (Pelechano et al 2007). Each of the 'people' making up a crowd needs to be individually modelled and persistent (at least over the duration of a scene); each individual needs to have a coherent and unique visual identity and a personal 'narrative' that they follow while being animated and rendered at the increasingly high frame-rates demanded by modern displays and users. A large number of approaches towards modelling agents' behaviour have been used from basic collision-avoidance with limited random choices of actions through to sophisticated agent models which communicate and engage in sophisticated social behaviours such as accompanying, following and queuing (Pelechano et al 2008).

\section{CROWDS IN IMMERSIVE MEDIA}

Crowds are comparatively rare in immersive media, and where they do occur, they have generally tended to be small and/or unresponsive. However, crowd sizes and realism have been improving and a small number of more recent games have incorporated sizable crowds either as a dynamic backdrop to the gaming environment or as a mechanism for scoring; success comes through 'pleasing' the crowd.

Hitman (2016) is a fully 3D stealth game and features over 300 non-playing characters (NPCs) with another 1,000 'Crowd NPCs in each scene, Hitman 2 (2018) has over 2,000 NPCs. The player can hide amongst and interact with crowd members who, in turn, react to events as they unfold around them. NPC behaviours are modelled using an Al decision-tree approach where each NPC character has their individual routines and goals and an awareness of their surroundings. 'Crowd' NPCs which have less computationally demanding control can be dynamically upgraded to full AI NPCs if the circumstances demand more activity and interaction. The intention of the developers was "to try and blur the line between the crowds and the behaviour-tree Al as much as possible" (Thompson 2019).
Project Highrise (2016) is a simulation game where the objective is to build and manage high rise towers, success comes from having satisfied tenants. The game is rendered in 2D. The extent of individual narratives for the NPCs is not completely clear but the makers claim to be able to support 1000 NPCs at 60fps (Zubick 2017). Planet Coaster (2016) is also a simulation game but it is rendered in stylised 3D and the player's task is to construct a theme park. The crowd system is much more ambitious with up to 10,000 individual, animated characters being supported by the game system. The developers were keen to produce believable crowd behaviours:

\begin{abstract}
"One thing I was particularly invested in was bringing the atmosphere of a real crowd into our virtual world, and in making each park guest aware of their surroundings. I wanted to capture little moments like walking by an entertainer doing silly things and seeing other guests watching and reacting." (McCarthy 2017)
\end{abstract}

One of the key characteristics of most current immersive VR experiences is their separation from the real world (Van Kerrebroeck et al 2017). This is partly the highly intrusive and isolating nature of the physical hardware required (wrap-around headset, headphones, perhaps a tether to a host computer) and also perhaps a recognition that the medium's affordances for transcendence, for placing the user in settings which are not those of everyday lived reality, are too powerful and tempting for most creatives not to explore. This removal or weakening of social context has its dangers however, it can limit the capacity for such works to be meaningful, to have resonances for the user that persist and transfer from the immediate 'in world' encounter to wider experiences of the world. It could also limit the capacity for such systems to reproduce the social experience of crowds which is no longer available in our 'new normal'. The current project seeks to counter this by providing each of the agents making up a virtual crowd with an inner narrative and appearance based on recent tweets and their authors.

\section{EMBODYING SOCIAL MEDIA, THE WISDOM OF CROWDS}

Generating a reasonably large number of software agents is not, in itself problematic, the challenge comes in achieving sufficient heterogeneity in appearance and character. These qualities can be generated by rules-based systems and Al agents but both approaches tend to be less useful when the crowd's context is not goal-directed or fixed in advance. A visitor to a theme park is easier to model 
convincingly than an individual in a crowd discussing the issues of the day. Using real-time data drawn from Twitter is a way to produce flexibility and wider social context to the members of a crowd in an immersive environment.

The current project emphasises the production of social context by conceiving of the 'crowd' as an oracle - a group of people presenting a variety of viewpoints on any topic the user wishes which are juxtaposed (in non-aggressive ways). Twitter is a platform that perhaps prioritises polemics, this feature was embraced, and each crowd member is provided with a placard with the tweet written on it.

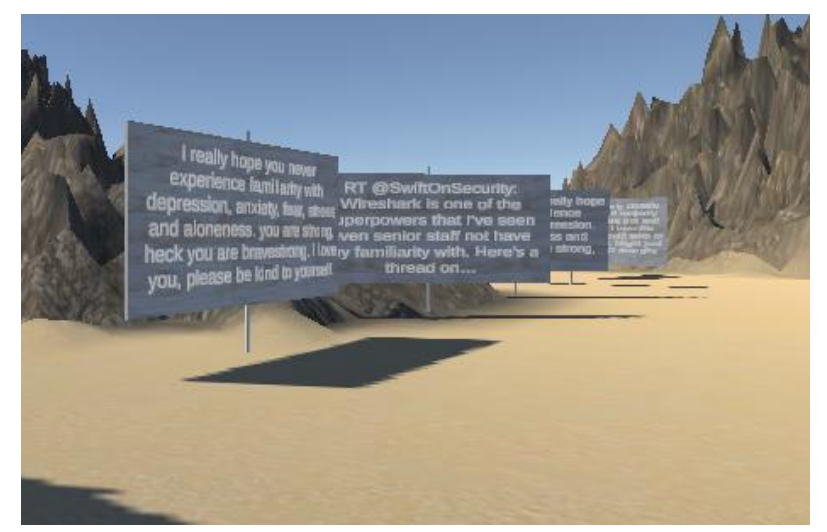

Figure 2: Placards with real-time text from a twitter search

Each tweet brings with it a surprisingly large amount of public metadata about the tweet itself and the person who made it (Perez 2010). This data can include the user's name, location, language, profile image and website URL. In an early version of the project, profile images were downloaded and applied to face masks to individualise agents. The customisation of characters will be increased to produce greater variation in both appearance, movement and voice, all based on metadata accompanying tweets.

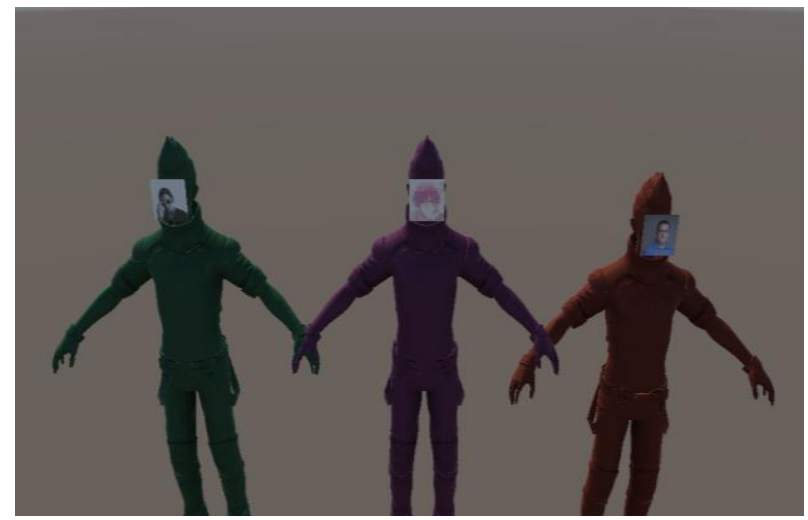

Figure 3: Early version of characters showing 'faces' sourced from Tweet authors

\section{CONCLUSION}

Crowd simulation is still a developing field with many applications which has seen significant advances in both fidelity and performance. Creating highly individual, persistent narratives for the members of these crowds in open situations is still a challenge and the use of real-time social media data is suggested as a useful approach. Future work could include the use of machine learning to match names and other metadata to appropriate characteristics which could be used to further individualise the generated agents.

\section{REFERENCES}

Dickinson, P., Gerling, K., Hicks, K., Murray, J., Shearer, J. \& Greenwood, J. (2019) Virtual Reality Crowd Simulation: Effects of Agent Density on User Experience and Behaviour. Virtual Reality, 23 (1) March, pp. 19-32.

Filingeri, V., Eason, K., Waterson, P. \& Haslam, R. (2017) Factors Influencing Experience in Crowds The Participant Perspective. Applied Ergonomics, 59 March, pp. 431-441.

Frontier Developments (2016) Planet Coaster [Computer game], Frontier Developments. Cambridge.

IO Interactive (2016) Hitman [Computer Game], Square Enix, Tokyo.

IO Interactive (2018) Hitman 2 [Computer Game] Burbank, Warner Brothers Interactive Entertainment

McCarthy, O. (2017) Game Design Deep Dive: Creating Believable Crowds in Planet Coaster. Gamasutra, 4 January [Online blog]. Available from:

<https://www.gamasutra.com/view/news/288020/G ame_Design_Deep_Dive_Creating_believable_cro wds_in_Planēt_Coaster.php $>$ [Accessed 17 March 2021].

Moore, S. (2020) What Am I Missing Thanks to Coronavirus? Crowds, Smiling at Strangers - and a Government That Cares. the Guardian [Online], Available from: <http://www.theguardian.com/commentisfree/2020/ aug/24/what-am-i-missing-thanks-to-coronaviruscrowds-smiling-at-strangers-and-a-governmentthat-cares> [Accessed 17 March 2021].

Pelechano, N., Allbeck, J. \& Badler, N. (2007) Controlling Individual Agents in High-Density Crowd Simulation. Proceedings of the 2007 ACM SIGGRAPH/Eurographics Symposium on Computer Animation, August, pp. 99-108. 
Pelechano, N., Allbeck, J. M. \& Badler, N. I. (2008) Virtual Crowds: Methods, Simulation, and Control. Morgan \& Claypool Publishers, USA.

Perez, S. (2010) This Is What a Tweet Looks Like [Online]. ReadWrite. Available from: <http://readwrite.com/2010/04/19/this_is_what_a_t weet_looks_like> [Accessed 15 December 2014].

SomaSim (2016) Project Highrise [Computer Game] Leicester, Kasedo Games.

Thalmann, D. \& Musse, S. R. (2012) Crowd Simulation. Springer Science \& Business Media.

Thompson, T. (2019) The Al of Hitman (2016) [Online]. Gamasutra. Available from: <https://www.gamasutra.com/blogs/TommyThomps on/20190807/346425/The_Al_of_Hitman_2016.ph> [Accessed 13 March 2021].
Van Kerrebroeck, H., Brengman, M. \& Willems, K. (2017) Escaping the Crowd: An Experimental Study on the Impact of a Virtual Reality Experience in a Shopping Mall. Computers in Human Behavior, 77 December, pp. 437-450.

$\mathrm{Xu}$, Ming-Liang, Hao Jiang, Xiao-Gang Jin, and Zhigang Deng. (2014) 'Crowd Simulation and Its Applications: Recent Advances'. Journal of Computer Science and Technology 29 (5): 799811. https://doi.org/10.1007/s11390-014-1469-y.

Zubek, R. (2017) 1000 NPCs at 60 FPS [Chapter]. In: Rabin, S. ed., Game Al Pro 3: Collected Wisdom of Game Al Professionals. Taylor \& Francis, pp. 105-112. 